\title{
Kernos
}

Revue internationale et pluridisciplinaire de religion grecque antique

$11 \mid 1998$

Varia

\section{La formation de Franz Cumont d'après sa correspondance (1885-1892)}

\section{Corinne Bonnet}

\section{OpenEdition \\ Journals}

Édition électronique

URL : http://journals.openedition.org/kernos/1232

DOI : 10.4000/kernos.1232

ISSN : 2034-7871

Éditeur

Centre international d'étude de la religion grecque antique

Édition imprimée

Date de publication : 1 janvier 1998

ISSN : 0776-3824

Référence électronique

Corinne Bonnet, «La formation de Franz Cumont d'après sa correspondance (1885-1892) », Kernos [En ligne], 11 | 1998, mis en ligne le 21 avril 2011, consulté le 22 avril 2019. URL : http:// journals.openedition.org/kernos/1232; DOI : 10.4000/kernos.1232 


\title{
La formation de Franz Cumont d'après sa correspondance (1885-1892)
}

\author{
Pour Francesco, «mon petit Franz» de six ans \\ à sa première année de formation
}

L'année 1997 a en quelque sorte été l'« année Cumont ». Plusieurs événements ont marqué le cinquantenaire de sa mort (20/8/1947), qui ont contribué à relancer l'intérêt de la communauté scientifique pour la vie et l'œuvre de celui qu'on s'accorde à considérer comme un historien majeur du $\mathrm{xx}^{\mathrm{e}}$ siècle. Un cycle de conférences et un Colloque International sur " Les syncrétismes » à l'Academia Belgica de Rome, une Table Ronde sur « Franz Cumont et la science de son temps » à Paris, la publication du premier fonds de sa correspondance scientifique ${ }^{1}$ et de diverses études le concernant ${ }^{2}$, la réédition d'Astrology and Religion among the Greeks and the Romans ${ }^{3}$ en sont autant de témoignages. Cette renaissance du débat culturel autour de l'auteur des Religions orientales dans le paganisme romain (1906, $4^{\mathrm{e}}$ éd. 1929) et de Lux Perpetua (posthume, 1949) a permis d'entamer une réflexion critique et nuancée sur son apport méthodologique et thématique aux sciences de l'Antiquité durant les 60 années de son activité d'historien (18871947) et sur sa place dans le " paysage » intellectuel et scientifique de son temps.

1 C. Bonnet (éd.), La correspondance scientifique de Franz Cumont conservée à l'Academia Belgica de Rome, Bruxelles-Rome, 1997 (Institut bistorique belge de Rome, Études de philologie, d'archéologie et d'bistoire anciennes, XXXV) (désormais cité La correspondance).

2 R. TurCan, Franz Cumont fondateur, in Hieros, 2 (1997), p. 11-20; C. BonNET, Franz Cumont et l'exégèse iconographique, ibidem, p. 21-30; on ajoutera les études sous presse de EADEM, Les lettres de René Dussaud à Franz Cumont conservées à l'Academia Belgica de Rome, in Festschrift Oswald Lorelz, Münster, 1998; La correspondance scientifique de Franz Cumont. Bilan et perspectives d'une recherche, in Les syncrétismes religieux dans la Méditerranée antique. Actes du Colloque à l'occasion du cinquantenaire de la mort de Franz Cumont, Academia Belgica, Rome, 25-27/9/1997, Bruxelles-Rome (Coll. de l'IHBR); De l'bistoire religietuse à l'bistoire des religions: les relations de Franz Cumont avec quelques bistoriens des religions, in Actes de la Table Ronde "Franz Cumont et la science de son temps", Paris, 5-6/12/1997, Paris-Rome (MEFRM).

3 A. Panaino (éd.), Franz Cumont, Astrologia e religione presso i Greci e i Romani (trad. P. Dalla Vigna), Milano, Mimesis, 1997 (avec Préface d'A. Panaiano, p. 9-46). 
Dans ce cadre, l'étude et la publication de la correspondance scientifique de Franz Cumont revêt une importance particulière. Ce riche ensemble constitue, comme je l'ai montré ailleurs, un témoignage de première main sur l'environnement au sein duquel il a évolué. Je me propose de préciser ici ce que la correspondance nous apprend des années de formation de Franz Cumont (né le $3 / 1 / 1868$ ), soit de 1885 , l'année des premières lettres conservées, à 1892, date de son engagement comme Chargé de cours à l'Université de Gand et par conséquent du début de sa carrière universitaire. Je me dispenserai de repréciser ici dans le détail ce en quoi consiste la correspondance scientifique de Franz Cumont, puisque je l'ai fait en plusieurs occasions $^{4}$. De même, pour l'établissement des points de repères biographiques, je renvoie le lecteur à mon Introduction sur La vie et l'œuvre de Franz Cumont, dans le volume à peine publiés. Celui-ci, je me dois de le souligner, porte exclusivement sur le premier fonds de correspondance : un fonds qui est conservé à l'Academia Belgica et qui contient un peu moins de 2.000 lettres. Le second fonds, jadis conservé à Wanlin et déposé à Rome en septembre 1997 par Monsieur J.-F. de Cumont, le petit-neveu de Franz Cumont, pour en permettre l'étude, est riche de près de 10.000 lettres en cours de dépouillement. On peut donc compter sur un total de près de 12.000 lettres reçues par F. Cumont entre 1885 (il avait alors 17 ans) et 1947 (date de sa mort, à 79 ans) ${ }^{6}$. Pour les années 1885-95, à l'étude du premier fonds, s'est aujourd'hui ajoutée celle du second fonds ${ }^{7}$, ce qui permet de présenter ici, pour la première fois, une mise au point complète du point de vue heuristique sur les années de formation de Franz Cumont. J'espère de la sorte fournir une contribution originale à la compréhension des expériences scientifiques, humaines et esthétiques qui contribuèrent à la formation intellectuelle du jeune Franz Cumont, de 17 à 24 ans $^{8}$.

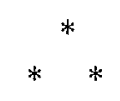

\footnotetext{
4 La correspondance, p. I-xil et les articles mentionnés à la n. 2.

5 La correspondance, p. 1-67.

6 Je rappelle que les lettres de la main de F. Cumont sont très rares dans les deux fonds (une quarantaine pour l'heure), mais que des recherches sont en cours pour retrouver celles qui sont conservées dans divers fonds d'archives européens et américains. Cf. un premier aperçu dans l'Appendice 3 de La correspondance, p. 513-525.

7 Le dépouillement s'accompagne de l'enregistrement pour chaque lettre d'une fiche informatisée. Il faudra sans doute environ quatre ans pour étudier l'ensemble du second fonds, une étude entamée en octobre 1997.

8 Je sais vivement gré à Monsieur André Motte d'avoir offert l'hospitalité de Kernos à ces quelques pages.
} 
D'entrée de jeu, quelques données chiffrées, sous forme de tableau ${ }^{9}$, pour mieux cerner le dossier sur lequel repose ma reconstruction.

$\begin{array}{ccc}\text { Année } & \text { Fonds I (Acad. Belg.) } & \text { Fonds II (Wanlin) } \\ 1885 & 0 & 1 \\ 1886 & 0 & 0 \\ 1887 & 1 & 3 \\ 1888 & 0 & 16 \\ 1889 & 2 & 39 \\ 1890 & 0 & 43 \\ 1891 & 34 & 52 \\ 1892 & 44 & 16 \\ \text { TotAL } & 81 & 170 \\ \text { TotAL DES DEUX FONDS : } 251 \text { lettres } & \end{array}$

S'agissant d'une période de formation, elle a pour cadre divers lieux : Gand, l'Allemagne, la France, l'Italie et la Grèce, selon un itinéraire et une chronologie que nous allons à présent retracer sur la base des informations contenues dans les lettres. On s'efforcera aussi de préciser la personnalité et le rôle des correspondants les plus significatifs.

À 16 ans, en 1884, F. Cumont entre à l'Université de Gand pour y étudier la philologie classique et il devient docteur en philosophie et lettres en 1887 à l'âge de 19 ans. Ses maîtres furent essentiellement Charles Michel, Paul Thomas et Adolphe De Ceuleneer. Dès 1887, il publie sa première étude sur Alexandre d'Abonotichos, un épisode de l'bistoire du christianisme (dans les Mémoires de l'Académie, à Bruxelles) et établit ses premiers contacts scientifiques. Le 29 septembre 1887, Ernest Babelon du Cabinet des Médailles de la Bibliothèque Nationale de Paris lui envoie des renseignements sur une monnaie portant une représentation de Glycon, tandis que le 5 octobre, Paul Thomas, latiniste de l'Université de Gand'11, le remercie pour l'hommage de son étude et ajoute :

Vous avez donc fait le premier pas dans la carrière scientifique! J'espère que vous ne vous en tiendrez pas là. Courage ! Continuez à travailler; vous pouvez rendre service à la science. Les études désintéressées sont malheureu-

9 J'ai pris en considération toutes les lettres, même celles dont la datation est incertaine et dont le correspondant est inconnu ou incertain (d'où une légère différence par rapport à la concordance chronologique fournie à la fin de La correspondance, p. 543, où seules 33 lettres figurent pour l'an 1891).

10 Je fais remarquer en passant que la répartition des lettres entre les deux fonds ne semble répondre à aucun critère logique.

11 Sur P. Thomas, cf. La comespondance, p. 450-454. 
sement trop négligées en Belgique ${ }^{12}$. C'est une vive satisfaction pour tous ceux qui ont à coeur l'avenir intellectuel et la réputation de notre pays, de voir des jeunes gens bien doués et pleins d'ardeur se consacrer aux recherches d'érudition.

En 1888, F. Cumont obtient le titre de candidat en droit à Gand et participe au concours des bourses de voyage du Ministère belge de l'éducation nationale avec un mémoire intitulé Sur la propagation des mystères de Mitbra : un thème dont il allait s'occuper pendant plus d'une décennie; en 1888, il publie aussi quatre articles scientifiques dans des revues belge et française. L'un d'eux lui vaut, le 7 juin 1888, une lettre du grand Salomon Reinach $^{13}$ :

Monsieur,

Votre travail sur les Dii aeterni ${ }^{14}$ était fort bon et votre correction est très plausible.

L'année 1888 est aussi celle où $F$. Cumont noue les premiers contacts épistolaires avec des savants allemands. Le philologue et historien Otto Seeck $^{15}$ lui écrit le 8 juin 1888 pour approuver l'interprétation qui a déjà obtenu l'adhésion de S. Reinach, tandis que les 11 et 29 juin, c'est au tour de Georg Wissowa ${ }^{16}$ de manifester son approbation et d'encourager F. Cumont à insister dans ses recherches sur les cultes étrangers :

je dunkler noch das ganze grosse Gebiet der im römischen Kulturgebiete recipirten Fremd Kulte ist, mit um so grösserer Freude ist jeder Beitrag zur

12 L'idée de "désintéressement " se retrouve dans une lettre du 14 août 1887 d'Armand Brifaut, un ami de F. Cumont : «Ce qui me plaît en vous, mon cher Ami, c'est votre amour désintéressé de la science, en un âge où tant d'autres subordonnent toute étude aux questions d'application pratique ». Juste avant, il écrit : «votre douceur pénétrante a exercé sa séduction sur moi, \& je dois vous avouer que je serais fort triste si vous ne me conserviez pas un souvenir très affectueux ». Au-dessus de cette phrase, F. Cumont a écrit de sa main : « niais ou courtisan ».

13 Sur S. Reinach et ses rapports avec F. Cumont, cf. La correspondance, p. 410-413. Sur la base du premier fonds de correspondance, je faisais remonter leurs contacts à 1894; ils sont en fait bien antérieurs et datent de l'aube de la carrière scientifique de F. Cumont.

14 Cf. Les dieux éternels des inscriptions latines, in RA, 11 (1888), p. 184-193.

15 À son sujet, cf. E. PATlagean, Dans le miroir, à travers le miroir: un siècle de déclin du monde antique, in Les études classiques aux XIX et XXe siècles : leur place dans l'bistoire des idées, Genève, 1979 (Entretiens sur l'Antiquité classique, XXVI), p. 209235, en part. p. 227-228 (élève de Mommsen, personnage très influent).

16 Sur G. Wissowa et ses contacts avec F. Cumont, cf. La correspondance, p. 478-480 (première lettre du premier fonds en 1892). Voir aussi BONNET, De l'bistoire religieuse à l'bistoire des religions, cit. (n. 2), sous presse. Dans sa carte postale du 29 juin, il définit «sehr interessanten und soliden » l'article sur les dieux éternels. 
Aufhellung des Dunkels zu begrüssen, zumal wenn er, wie der Ihrige, ein so klares Resultat zu Tage fördert.

Il voit d'un œil très favorable l'idée de reprendre l'étude de Mithra et il espère utiliser les résultats obtenus pour ses propres travaux. À n'en pas douter, ce contact dut être très important pour le jeune $F$. Cumont qui cherchait alors sa voie ${ }^{17}$. En septembre $1888, F$. Cumont se prépare à partir pour l'Allemagne et, le 26 octobre, Wissowa se réjouit de sa présence en Allemagne qui lui donnera sans doute l'occasion de le rencontrer à Marburg où il enseigne. $\mathrm{F}$. Cumont pour sa part réside à Bonn ${ }^{18}$ où il va fréquenter une série de cours à l'Université durant le «Wintersemester »1888-89. L'agenda académique de $\mathrm{F}$. Cumont, retrouvé dans ses archives conservées à l'Academia Belgica de Rome, m'a permis d'établir la liste probable de ses professeurs : parmi eux, citons Nissen, Kekulé von Stradonitz, Lübbert, Bücheler, Prym $^{19}$ et surtout Hermann Usener ${ }^{20}$, l'animateur du Bonner Kreis, un très important foyer intellectuel allemand, qui eut assurément une influence considérable sur F. Cumont et l'orientation de ses recherches. À lire une lettre de Ch. Michel, du 13 novembre 1888, l'on comprend combien F. Cumont était enthousiaste de cette expérience, mais son maître gantois redoutait qu'il ne se germanisât trop :

Mon cher Ami,

Grand merci de votre longue et bonne lettre, elle m'a vivement intéressé et je suis bien heureux de vous voir content là-bas. Puissiez-vous ne pas revenir trop germanisé ! Il est vrai que moi aussi j'ai connu l'enthousiasme pour l'Allemagne et que j'en suis bien revenu.

Je suis charmé d'apprendre qu'Usener a été aussi aimable, mais je ne me figure pas du tout que c'est à ma lettre que vous devez cet accueil. Mithra et vos travaux antérieurs ont bien plus fait pour cela que mes quelques lignes. Gloria donc Deo Invicto Mithrae.

La lettre de $\mathrm{P}$. Thomas, du 3 décembre 1888, est plus positive encore :

Cher Monsieur,

(...) Les détails que vous me donnez sur votre séjour à Bonn m'ont été fort agréables. Je ne doute pas que vous tiriez le plus grand profit des leçons des

17 Dans une lettre successive, du 9 janvier 1889 , à propos d'un autre article, Wissowa exprime son appréciation concernant «Fragestellung und Methode ».

18 La carte postale de Wissowa est adressée «Hofgartenstrasse 1 » à Bonn.

19 Une lettre de Ch. Michel nous apprend que F. Cumont était le seul étudiant du cours de syriaque de Prym.

20 Cf. La correspondance, p. 7-10. Sur la personnalité d'Usener et ses rapports avec F. Cumont, $c f$. ibidem, p. 459-462 et De l'bistoire religieuse à l'bistoire des religions, cit. (n. 2), sous presse. Comme je l'ai signalé dans $L a$ correspondance, p. 6, l'existence d'un manuscrit du cours de Mytbologie d'Usener de 1886-87 de la main de F. Cumont dans ses archives prouve qu'il fréquenta ce cours dès l'époque de ses études à Gand. 
professeurs, de la fréquentation des musées, etc. Vous devez vous sentir là-bas dans une autre atmosphère scientifique qu'ici; vous avez déjà pu constater la liberté qui règne dans les études universitaires, la spontanéité et l'initiative des étudiants, la passion des <des> découvertes et du progrès qui anime le corps professoral. Au premier abord, on est un peu dépaysé au milieu de tous ces cours, de toute cette variété de sujets, de toute cette organisation si différente de la nôtre. Mais on s'y fait bientôt, et l'on se sent à l'aise dans ce grand atelier de science.

Fin janvier, le semestre hivernal arrive à son terme et $\mathrm{F}$. Cumont se prépare à quitter Bonn pour Berlin, un autre " grand atelier de la science », selon la belle expression de P. Thomas : le transfert dut se faire en mars. Les contacts scientifiques en cours se poursuivent: de nouveaux encouragements lui arrivent de G. Wissowa, qui attend sa visite à Marburg en mars; Ch. Michel le tient au courant du concours des bourses de voyage (ce que fait aussi son père) et des affaires gantoises; P. Thomas s'informe de ses projets scientifiques relatifs à Julien ${ }^{21}$. De nouveaux correspondants s'ajoutent aux premiers, qui témoignent de la réputation croissante du jeune savant belge. Ainsi, le 15 mars 1889, F. Cumont est-il le destinataire d'une lettre de Jean Réville (1854-1908) ${ }^{22}$, fils du théologien protestant (et libéral) et historien des religions Albert Réville (1826-1906), le premier titulaire d'une chaire d'Histoire comparée des religions au Collège de France. J. Réville était quant à lui Professeur à la Ve section («sciences religieuses ») de l'École Pratique des Hautes-Études depuis 1886, année au cours de laquelle il publia son célèbre ouvrage sur La religion à Rome au temps des Sévères, et il succéda à son père au Collège de France en 1907, peu avant de mourir, laissant la place à Alfred Loisy. Il devint aussi, en 1884, le Directeur de la Revue de l'bistoire des religions, fondée en 1880 par un autre protestant, Maurice Vernés. C'est précisément pour lui demander un article sur le mithriacisme ${ }^{23}$ qu'il écrit à F. Cumont :

\section{Monsieur,}

J'ai remarqué à plusieurs reprises des articles ou des notices, émanant de vous, sur la religion mithriaque. La Revue de l'Histoire des Religions, dont la direction m'est confiée, serait le recueil scientifique le plus approprié à la

21 Un fascicule de 31 pages intitulé Sur l'autbenticité de quelques lettres de Julien, sera en effet publié dans les collections de l'Université de Gand en 1889. Une lettre d'Henri Pirenne, du 18 mai 1889, confirme l'acceptation officielle du manuscrit.

22 Cf. P. Alphandéry, J. Réville, in RHR, 57 (1908), p. 269-282; E. De Faye, L'cuure bistorique et scientifique de M. Jean Réville, ibidem, p. 283-292. J. Réville fit un cycle de conférences au Musée Guimet notamment sur le mithriacisme, mais sa perpective était surtout celle d'un historien du «christianisme syncrétiste ». Sur le contexte intellectuel et le rôle des protestants, cf. Fr. LAPLANCHE, La Bible en France entre mythe et critique XVI XIX $X^{e}$., Paris, 1994.

23 Je ne sais comment F. Cumont répondit à J. Réville, mais aucun article de lui ne parut dans la $R H R$ avant 1901, et ce ne fut pas sur Mithra. 
publication de vos études ultérieures sur le Mithriacisme. Je prends donc la liberté de vous demander si vous seriez disposé à nous donner quelques travaux sur ces questions que vous connaissez si bien et auxquelles je porte un intérêt tout particulier.

Je ne puis malheureusement pas joindre à cette invitation la promesse d'une rémunération pour les articles que vous nous donneriez. Nous travaillons tous gratuitement. Mais il en est de même dans presque toutes les revues d'ordre purement scientifique, en sorte que j'espère que ce ne serait pas là un motif préalable pour nous refuser votre concours.

Je sais aussi que vous n'êtes pas d'accord avec moi sur plusieurs points que j'ai traités dans ma Religion à Rome sous les Sévères. Mais il va sans dire que je n'ai pas la prétention d'imposer à tous les collaborateurs de notre Revue les opinions ou les solutions que j'ai soutenues moi-même. Vous jouiriez de la plus parfaite liberté pour attaquer mes idées sur la propagation du mithriacisme et pour me corriger. Justement parce que j'ai étudié le sujet par moi-même, je sais combien il est complexe, combien il est encore insuffisamment connu sur bien des points et combien certaines assertions énoncées dans mon travail sont sujettes à caution.

Au nombre des nouveaux correspondants, on signalera évidemment Theodor Mommsen (1817-1903) ${ }^{24}$. Le 2 avril 1889, ce «monstre sacré », alors âgé de 72 ans, adresse un bref billet en allemand ${ }^{25}$ au jeune F. Cumont de 21 ans pour le convier à passer une soirée en famille, chez lui, le 6. Le milieu berlinois devait être des plus stimulants, et Monsieur Cumont père ne s'y trompe guère qui interroge inlassablement son fils sur les cours qu'il suit, les professeurs qu'il rencontre, les compagnons d'étude qu'il fréquente. Dès 1890, on rencontre aussi Hermann Diels (1848-1911) parmi les correspondants de F. Cumont : un grand savant qui joua un rôle essentiel dans sa formation et dans ses rapports avec l'Allemagne ${ }^{26}$. F. Cumont, de son côté, reste en contact avec ses compagnons du «Bonner Kreis »: plusieurs d'entre eux, comme Hermann Joachim, Richard Heinze, François Chavannes, Hugo Rabe, Otto Kern, George Lincoln Hendrickson et Otto Cuntz lui écrivent régulièrement pour l'inviter à Bonn, pour le tenir au courant de leurs travaux, pour lui donner des nouvelles du "Maître », Usener, pour s'informer de ses impressions berlinoises. Bien des amitiés de cette époque, à Bonn surtout, mais aussi à Berlin, alimentèrent la correspondance de F. Cumont pendant plusieurs décennies, parfois jusqu'aux dernières années de son existence. Ces lettres nous livrent quelques détails sur les occupations de F. Cumont, par

24 À son sujet, cf. La correspondance, p. 346-350.

25 Les autres lettres de Mommsen du premier fonds sont toutes en français; elles ont fait l'objet d'une publication par F. Vercauteren, Une correspondance scientifique Théodore Mommsen - Franz Cumont (1894-1901), in BAB, 40 (1954), p. 68-90.

26 Sur H. Diels et ses rapports avec F. Cumont, cf. La correspondance, p. 169-177. Il y a aussi de très nombreuses lettres de lui dans le second fonds de correspondance. Il fut le promoteur de l'élection de F. Cumont à l'Académie de Berlin en 1911 ( $\mathrm{c}$. La correspondance, p. 7, n. 18). 
exemple un projet de voyage à Dresde et Leipzig, en juin 1889. Le semestre terminé, $\mathrm{F}$. Cumont décide de prolonger son séjour à Berlin pour continuer à travailler avec Diels, nonobstant une certaine opposition de son maître gantois, Ch. Michel qui lui écrit le 10 juillet 1889 :

Mon cher Ami,

(...) J'ai beaucoup pensé à vous (mes remords me tourmentaient souvent) et à votre intention de passer encore un semestre à Berlin, et pour ma part, je ne vous y engage pas. Évidemment, ce ne serait pas du temps perdu, vous sauriez utiliser ce séjour et il y a toujours à apprendre. Mais il me semble que vos projets de voyage étaient fort beaux et fort utiles aussi, et puisque vous seriez obligé de les écourter pour rester à Berlin, je ne puis m'empêcher de trouver que ce serait grand dommage. $M^{r}$ Thomas m'a parlé d'un travail que vous avait indiqué Diehls (sic) et qui serait intéressant. Mais ce ne seront jamais les sujets de travaux qui vous manqueront, puisque vous en avez déjà trouvé plusieurs tout seul. Et Diehls n'est pas le seul homme qui pourra vous guider, jusqu'au moment prochain où vous n'aurez plus besoin de guide.

Voilà mon avis puisque vous me le demandez: mais notez bien que si vous ne le suivez pas, je ne vous en voudrais nullement. Vous êtes mieux placé que moi pour juger de la situation, et je ne voudrais pas avoir sur la conscience de vous avoir détourné d'un bon travail et d'un utile semestre.

De cette lettre ressort combien la personnalité de Diels a impressionné F. Cumont qui décide effectivement de passer un second semestre, le semestre hivernal 1889-90, à Berlin, après avoir passé une partie de l'été à Wanlin. Ch. Michel lui-même, dans une lettre du 19 août, lui écrit : « je puis vous dire que j'ai été converti à l'idée de vous voir passer l'hiver à Berlin : les raisons que vous donnez sont très sérieuses ». En accord avec $\mathrm{P}$. Thomas, il lui propose aussi des conseils pour l'« après-Berlin »: aller de suite à Paris et se rendre ensuite à Athènes et Rome, mais F. Cumont fit l'inverse. En septembre 1889, F. Cumont est à nouveau à pied d'œuvre à Berlin : le 5 septembre, Otto Hirschfeld se réjouit de son retour. Son père continue à le tenir au courant des questions familiales et ses maîtres, des affaires gantoises. Par une lettre de son père du 19 novembre 1889, nous apprenons que $\mathrm{F}$. Cumont continuait à fréquenter la maison de Mommsen, tandis que, dans une lettre du 4 décembre, $M$. Cumont père fait allusion à deux projets de son fils: s'occuper des manuscrits de Philon le Juif ${ }^{27}$ et se rendre à Vienne.

Dès la fin de l'année 1889, des projets concernant F. Cumont sont élaborés à Gand. En témoigne une lettre de P. Thomas, du 17 décembre :

Cher Monsieur,

Le moment est venu de vous faire prendre pied à l'Université de Gand. Je suis accablé de besogne; j'ai besoin d'un assistant ou d'un suppléant. J'ai soumis la question à $M$. Wagener et à $M$. Wolters, qui m'ont complètement approuvé; je vous ai proposé, et cette proposition a été accueillie avec la plus

27 Il en résultera le volume Pbilonis de aeternitate mundi, paru à Berlin en 1891. 
grande faveur. Je me suis rendu hier à Bruxelles pour avoir à ce sujet un entretien avec le Ministre - sans prendre d'engagement formel, il m'a dit qu'il était tout à fait disposé à faire droit à ma demande. Je viens maintenant, avant de faire la démarche officielle et décisive, vous demander votre assentiment à la combinaison indiquée.

J'espère que vous ne me le refuserez pas. Vous connaissez l'amitié et l'estime que j'ai pour vous; je vous prie d'avoir confiance en moi et de me laisser faire. Il y a urgence - l'occasion est là; ne la laissez point échapper. Si vous hésitiez, vous nous mettriez dans un cruel embarras; car il y a des candidats éventuels dont nous ne voulons à aucun prix, et, s'ils avaient vent de la chose, ils mettraient tout en cuvre pour réussir. Dieu sait alors ! ... Acceptez, et vous rendrez un grand service à l'Université.

P. Thomas propose à F. Cumont de n'avoir aucune attribution fixe afin d'être libre durant un semestre pour se rendre en Italie et en Grèce. Sans réponse, $\mathrm{P}$. Thomas renouvelle sa demande le 24 décembre, tandis que le 25 , c'est au tour de Ch. Michel de vanter les mérites de ce projet : « je ne saurais trop pour ma part vous engager à vous laisser faire ». Il espère obtenir aussi l'engagement de Léon Parmentier, qui vient de présenter un « très brillant doctorat spécial ${ }^{28}$.

F. Cumont décline cependant l'offre: pour lui, le moment n'est pas encore venu et sa formation à l'étranger n'en est qu'à son début : il n'entend pas la sacrifier. Ce n'est finalement qu'en 1892 qu'il entre à l'Université de Gand, après de nombreuses sollicitations et malgré de fortes résistances de sa part. F. Cumont, comme il résulte d'une lettre de P. Thomas, du 19 janvier 1890, suggéra plutôt d'engager Parmentier et il fut si habile dans sa réponse qu'il accrut encore la considération dont il jouissait auprès de ses maîtres; voici ce qu'écrit P. Thomas :

\section{Cher Monsieur,}

(...) P.S. Je tiens à vous dire que votre refus d'entrer actuellement à l'Université et les considérations élevées que vous avez fait valoir pour le motiver ont fait la meilleure impression et ont augmenté encore l'estime et la sympathie dont vous jouissez parmi nous. Le Ministre m'a dit textuellement ceci :"Quand M. Cumont n'aurait d'autre titre que la conduite qu'il a tenue en cette circonstance, je serais heureux de le nommer dès que le moment sera venu". Inutile de vous déclarer que, malgré le regret que j'ai éprouvé quand vous avez décliné l'offre que je vous faisais, je loue sans réserve votre désintéressement et votre amour de la science. Si tout le monde vous ressemblait !....

En février, F. Cumont se prépare à rejoindre la Belgique pour se rendre ensuite à Vienne dont les «plaisirs trop faciles » semblent " fort dangereux » aux yeux de son grand-père maternel, Charles Faider qui, dans deux lettres du 9 janvier et du 12 février 1890, dit approuver sa décision relative à Gand et se

28 Sur L. Parmentier et ses rapports avec F. Cumont, cf. La correspondance, p. 371-383. 
réjouir de ses activités berlinoises, notamment de ses «conférences avec Mommsen ». Le 12 février encore, Ch. Michel lui annonce " une nouvelle tentative pour vous avoir » [scil. : à Gand] et l'engage à accepter : en vain. Début avril, F. Cumont est à Munich, étape de son voyage à Vienne, et le 15 avril, une lettre de son père nous apprend qu'il s'est installé dans la capitale autrichienne. Il projette un voyage en Transylvanie sur les pas de Mithra qui occupe une bonne partie de son séjour autrichien.

Du 4 mai 1890 date la première lettre d'H. Diels à $F$. Cumont. Il remercie son disciple de lui avoir écrit de Vienne et se réjouit qu'il travaille sur Philon; il lui fournit une série de conseils pour l'index de son étude ${ }^{29}$ et propose d'en relire le manuscrit. Sa lettre se termine par l'expression d'un souhait qui dit bien la profondeur du lien entre les deux hommes : «Vielleicht kommen Sie wieder zu uns, um mir als Iolaos beizustehen ».

F. Cumont espérait une visite de sa mère à Vienne, mais elle ne se concrétisa pas. Son père lui écrivait en tout cas très régulièrement et l'entretenait notamment de l'évolution de la situation à Gand; le 20 mai, voici ce qu'il en dit :

Mon cher Frantz,

(...) Il (= Wagener, l'administrateur de l'Université de Gand) m'a dit, ce que tu sais déjà, qu'il se chargera de divers cours, \& que d'autres professeurs en feront autant, pendant un an ou dix-huit mois, jusqu'à ce que tu puisses prendre tes cours.

On ne peut manquer d'être surpris par la « cour» que les professeurs gantois font à un jeune savant de 22 ans que l'on attend à bras ouverts, même s'il n'est pas sûr qu'on pourra lui offrir de suite le titre de Professeur plutôt que celui de Chargé de cours. En juillet 1890, alors que son séjour viennois s'achève, $C h$. Michel et $P$. Thomas ne manquent pas de se manifester à nouveau; le second se félicite des recherches de F. Cumont sur Philon et sur Julien, mais, à propos de Mithra, il est fait allusion à un F. Cumont inattendu, prêt à abandonner sa recherche :

Cher Monsieur,

(...) Quant à Mithra, je ne vous blâme pas de renoncer à l'achever si un travail d'ensemble exige tant de temps et de peine pour un mince profit.

Les vacances estivales ont à nouveau pour cadre Wanlin : les lettres du mois de septembre 1890, provenant d'amis allemands, comme Otto Cuntz ou Otto Hirschfeld, font allusion à un prochain départ pour l'Italie et la Grèce. Une phase de la formation de F. Cumont s'est en effet conclue et une autre est sur le point de commencer, La première l'a profondément marqué sur le

29 Diels devait avoir une réputation particulière dans le domaine des index. A.D. Nock, dans une lettre à F. Cumont, du 9 novembre 1938, à propos des Mages hellénisés, loue la qualité de l'index en ces termes: «What a wonderful index you made ! Diels would have admired it, and one can say no more ». Cf. La correspondance, p. 362. 
plan scientifique et intellectuel, et les rapports de F. Cumont avec la science allemande furent toujours profonds et intenses. Parmi ses maittres, on peut certainement affirmer qu'une place de choix revient à Hermann Usener, pour ce qui concerne l'histoire des religions et l'histoire des sciences, l'astrologie en particulier, et à Hermann Diels, pour ce qui est de la philologie, de la philosophie et de la pensée religieuse. Mais on ne négligera pas l'apport d'autres grandes personnalités, comme Mommsen, Hirschfeld, Robert, Benndorf, Krumbacher, Kekulé, dans les domaines du droit, de l'épigraphie, de la byzantinologie, de l'histoire de l'art, etc. F. Cumont apprit en tout cas à faire de l'histoire sans frontière et à évoluer avec aisance et sûreté dans les vastes espaces de l'Altertumswissenschaft. Refusant une fois encore de suivre les conseils de ses maîtres gantois, il choisit de se soumettre ensuite au "voyage initiatique » en Italie et en Grèce, avant de reprendre sa formation érudite à Paris. À Rome, à Athènes, et dans l'univers méditerranéen en général, F. Cumont était certes à la recherche des traces tangibles de l'époque sur laquelle il travaillait, mais au-delà du regard du spécialiste, du technicien, - il y avait aussi la dimension esthétique, poétique, des émotions, à laquelle du reste plusieurs lettres font allusion. Enfin, ces voyages allaient être aussi l'occasion d'entrer en contact avec d'autres collègues.

Fin septembre, O. Hirschfeld se propose de l'introduire auprès de Petersen et Hülsen à Rome, tandis qu'H. Diels lui demande de transmettre ses salutations à $O$. Kern à Athènes. L'itinéraire de $F$. Cumont ne nous est pas parfaitement connu : il se rendit apparemment à Trieste, ville natale de son grand-père Charles Faider, puis à Venise, Corfou et enfin Athènes, mais nous ignorons la date de son arrivée. Une lettre de son père, du 20 novembre 1890, nous fait partager l'émotion que suscita en lui la visite de l'Acropole :

Mon cher Frantz,

(...) Je vois que tu es dans un enthousiasme croissant au sujet de l'acropole; c'est dommage que tu ne sois pas arrivé à Athènes par mer; l'aspect est encore bien plus frappant. Je crois que c'est la plus belle chose qui ait jamais été construite où que ce soit, \& l'on se figure ce que cela devait être aux jours de la splendeur d'Athènes.

Dans une lettre du 2 décembre 1890, il est question d'un voyage que $F$. Cumont a fait en Étolie et d'une prochaine excursion dans le Péloponnèse. C'est armé d'un appareil photo que F. Cumont sillonnait la Grèce, puisque son père exprime le désir de voir les photos de ses voyages, mais malheureusement, nous ne les avons pas conservées ${ }^{30}$.

Fin décembre 1890, un événement dramatique interrompt le séjour de F. Cumont en Grèce : alors que, depuis plusieurs semaines, son père le tenait

30 On conserve en revanche à Rome un abondant matériel photographique relatif à son voyage d'exploration dans le Pont et en Petite Arménie en 1900; il a fait l'objet d'une exposition en septembre 1997, par les soins de Véronique Krings, qui travaille encore sur ce matériel. 
informé de l'état de santé préoccupant de son grand-père maternel, à l'improviste, c'est sa mère, Marie Faider, qui tombe gravement malade et meurt en l'espace de quelques jours. Les premières lettres de condoléances datent du 27 décembre 1890 et l'événement fut très durement ressenti par toute la famille Cumont. Monsieur Cumont père continua à écrire très régulièrement à son fils, mais il ne cacha jamais sa douleur et le regret profond qu'il éprouvait au souvenir de sa femme. F. Cumont revint précipitamment de Grèce pour assister aux funérailles de sa mère à Alost, mais il repartit illico, puisque le 14 janvier 1891, son père lui écrit en le sachant déjà arrivé à Rome. Toute la première moitié de l'année 1891 allait être italienne, riche de découvertes et d'expériences.

Le milieu romain est très international : $F$. Cumont y retrouve en effet une série de connaissances de sa période allemande et autrichienne. Mais Rome et l'Italie sont surtout, comme à l'époque du " grand tour » des artistes, un puits d'émotions esthétiques incomparables. Une lettre de sa sœur Lucie, du 3 février 1891, et une autre de Ch. Michel, du 3 mars, confirment qu'il en fut ainsi pour F. Cumont :

Mon cher Franz,

Nous avons été heureux de recevoir de tes nouvelles et de voir combien tu es émerveillé de ce que tu vois à Rome. J'ai éprouvé la même stupéfaction que toi, en arrivant place $S^{t}$ Pierre.

Mon cher ami,

(...) Il y a onze ans qu'à pareille date je parcourais comme vous avec un véritable enivrement cette ville de Rome dont j'ai conservé le souvenir le plus lumineux. Quelle joie de pouvoir ainsi jouir de tous les trésors et de tous le souvenirs accumulés dans cette ville incomparable.

Il paraît que les Italiens continuent à faire tout ce qu'ils peuvent pour enlever à Rome ce qui fait son cachet unique, mais quand j'y suis allé on disait la même chose depuis longtemps, et cependant, j'étais dans le ravissement de ce qui restait encore. Je suppose que vous en êtes là aussi.

Les premiers contacts avec le monde scientifique italien ont lieu de suite; dans une lettre du 5 février 1891, M. Cumont père se félicite de la collaboration de son fils au Dizionario di anticbità romane d'Ettore De Ruggiero ${ }^{31}$. D'après une lettre du même $M$. Cumont, en date du 24 février, nous apprenons que F. Cumont, qui prépare une excursion à Assise, rencontre certaines difficultés à obtenir les permis pour travailler dans les musées italiens. Tandis que fait sensation la nouvelle de la découverte de la Constitution des Athéniens d'Aristote, dont $\mathrm{M}$. Cumont parle à plusieurs reprises, $\mathrm{F}$. Cumont jouit de l'Urbs: cérémonie religieuse à la Chapelle Sixtine et visite de la Villa Adriana. Le 29 mars, son père fait allusion à un prochain départ pour Florence : son séjour à Rome aura donc duré environ trois mois. Le 8 avril, on

31 Sur cette collaboration, cf. La correspondance, p. 155-157. 
apprend par la même source qu'il va bientôt se rendre à Pérouse et sillonner la Toscane. Le 28 avril, F. Cumont est de retour à Florence et son père lui écrit :

Je n'ai jamais été à Pérouse, mais je savais que cette ville est très remarquable. Je ne m'étonne pas qu'après un séjour de trois mois à Rome Florence t'ai paru petite; c'est néanmoins une ville charmante et très intéressante; ne manque pas d'aller voir l'Académie des beaux-arts où l'on voit très bien les progrès successifs de la peinture depuis Cimabue. Je doute que tu aies fini à Florence en quinze jours, car il faut aller de là à Pise, à Sienne; on dit que Pistoja, Arezzo, \& d'autres endroits encore sont aussi fort intéressants; c'est chaque fois un jour; il faut aussi faire l'excursion à Fiesole.

Le 6 mai, F. Cumont est sur le point de quitter Florence pour Bologne, avant de se rendre à Naples. Le 23 mai, son père accuse réception d'une lettre où il est question de Bologne :

Mon cher Frantz,

J'ai reçu ta lettre de Bologne \& vois que tu n'es pas très enthousiaste de cette ville qui est cependant intéressante à voir (...); il est certain que tout cela est loin de valoir Florence, il n'y a pas de comparaison à faire entre les deux.

F. Cumont semble intentionné à s'arrêter à Pesaro et Assise, et son père lui rappelle Rimini et Foligno, en concluant : "si l'on voulait s'arrêter dans toutes les villes où il y a quelque chose à voir on n'aurait jamais fini ». Une lettre de Ch. Michel, du 1er juin 1891, annonce le retour de F. Cumont en Belgique pour juillet: il faut suivre la situation à Gand, où trois ou quatre toges devraient être créées; il sera opportun que F. Cumont obtienne une lettre de soutien d'H. Diels. Début juin, F. Cumont se rend à Naples et il prévoit, sur la route du retour vers la Belgique, de visiter Nîmes et Arles.

Naples, c'est aussi Pompéi. Par une lettre de M. Cumont du 19 juin 1891, nous apprenons que Franz y a passé quatre jours, trente-deux ans après que ses parents y furent allés. En revanche, il a décidé de renoncer à la Sicile :

Par ce que tu me dis je vois que les fièvres règnent maintenant en Sicile comme il y a quarante ans, \& je suis bien aise que tu aies renoncé à y aller; c'est un voyage qu'on doit faire en Mars ou en Avril \& pas en Juin.

Son père l'encourage par contre à visiter Avignon et Orange sur la route du retour. Apparemment, toutefois, F. Cumont dut changer ses programmes : fin juin, le 27, il est à Maros Nemethi, en Hongrie, chez le Comte Geza Kuun d'Osdola, et il se prépare à regagner la Belgique via Vienne. En juillet, la correspondance qui lui parvient en Belgique témoigne de la longévité des échanges scientifiques nés dans les mois et les années qui ont précédé; ainsi Otto Benndorf, qui fut son mâtre à Vienne, le remercie-t-il pour l'envoi de son fascicule sur Philon le 9 juillet 1891, tandis qu'H. Diels suit avec grande attention tous ses travaux. 
Le 30 jullet, $\mathrm{F}$. Chavannes, un compagnon d'études de Bonn et Berlin, lui écrit de Paris pour lui faire part de sa nouvelle expérience : contacts avec Haussoullier, Havet, Swoboda; F. Cumont se prépare à goûter lui aussi à la vie parisienne $^{32}$. Il quitte la Belgique pour la France au début du mois de novembre 1891 et s'inscrit le 13 novembre à la IV ection « Sciences Historiques et Philologiques » de l'École Pratique des Hautes-Études, comme cela résulte du registre de l'année académique 1891-1892, qui indique comme domicile « 50 , rue des Bernardins ${ }^{33}$ et comme Professeurs choisis « Haussoullier, Carrière, Duchesne, Psichari ${ }^{34}$. Parmi les compagnons d'étude de F. Cumont cette année-là à la $I^{2}$ section (mais pas nécessairement pour les mêmes cours), on relèvera les noms d'Alfred Boissier, Émile Boisacq, Émile Bourguet, Auguste Bréal, Louis Bréhier, Jean-Baptiste Chabot, Fernand Chavannes, Alfred Foucher, Pierre Jouguet, Louis de la Vallée Poussin (futur collègue de F. Cumont à Gand), François Lods, Ferdinand Lot, Paul Perdrizet et Louis Rougier, autant de savants en herbe dont certains furent longtemps les correspondants de F. Cumont.

B. Haussoullier ${ }^{35}$ donnait un cours intitulé « Épigraphie et antiquités grecques » que F. Cumont fréquenta assidument de la mi-novembre 1891 à la fin juin 1892; Auguste Carrière ${ }^{36}$ enseignait les "Langues sémitiques », en particulier le syriaque. $F$. Cumont suivit ce cours de manière plus irrégulière (il fut absent entre le 11 février et le 28 avril, alors qu'il participait aux cours d'Haussoullier et de Duchesne); Monseigneur Louis Duchesne ${ }^{37}$ avait pour domaine les «Antiquités chrétiennes »: F. Cumont fut un de ses auditeurs assidus; enfin, Jean Psichari était le titulaire du cours de «Philologie byzan-

32 Rares sont les lettres qui éclairent la vie privée de $\mathrm{F}$. Cumont durant cette période. On retiendra une lettre d'un compagnon d'études, $M$. Muret, le 10 juillet 1892, à propos d'une certaine Mariette qui lui demande de l'argent, mais dont il ne veut plus entendre parler : «Et puis, les femmes m'embêtent, j'en ai plein le dos; quand on voit où elles nous mènent, le mieux est encore d'en user le moins possible ". Et il ajoute : "Peux-tu croire que la maligne, pour me piquer au jeu, me raconte que tu lui as offert de l'argent, mais qu'elle a refusé ? ». Cette encombrante maîtresse (comme Muret la définit luimême) n'empêcha pas les deux hommes de rester amis très longtemps : Muret écrit encore F. Cumont en 1939 (sur la base du rer fonds).

33 On conserve dans les archives Cumont de l'Academia Belgica à Rome les notes de logement (« Maison meublée tenue par S. Bachet, 27 \& 50 rue des Bernardins, Paris, Service à volonté dans les chambres ») de F. Cumont à Paris, entre février et octobre 1892. Ces notes concernent, outre la location d'un appartement (au prix de 120,10 francs), la fourniture de charbon et de pétrole, ainsi que le blanchissage.

34 J'ai pu consulter ce registre (pour F. Cumont : folio 74) et celui des signatures à l'École Pratique, à Paris. Je remercie vivement le personnel de la bibliothèque de la IV section pour sa disponibilité et $M$. Maurice Sznycer qui a bien voulu m'y présenter.

35 Cf. La correspondance, p. 224-229.

36 Cf. La correspondance, p. 137-139.

37 Cf. La correspondance, p. 177-180. 
tine et néo-grecque » que F. Cumont ne suivit que de fin novembre à la mimars.

Ce milieu de l'École Pratique des Hautes-Études eut une grande influence sur F. Cumont et sur l'orientation de ses recherches. On notera d'emblée qu'il choisit de fréquenter la $I^{\mathrm{e}}$ section, celle des sciences historiques et philologiques, et non la $\mathrm{V}^{\mathrm{e}}$ section, celle des sciences religieuses (fondée en 1886). La formation vers laquelle il tendait était celle d'un parfait connaisseur et utilisateur de toutes les sources, surtout, dans cette phase en tout cas, des sources écrites. Partant de la philologie classique, il étend donc son horizon vers les époques plus récentes, époques byzantine et chrétienne, et vers les domaines voisins, en particulier le domaine sémitique ${ }^{38}$; enfin, bon connaisseur des textes littéraires, il se forge un bagage d'épigraphiste. Il ne semble nullement soucieux de se préparer à une carrière d'historien des religions, comme on l'entendait alors, c'est-à-dire en pratiquant le comparatisme. Le milieu. parisien lui donna encore l'occasion d'entrer en contact avec des savants importants ou en passe de le devenir; outre les noms de ses professeurs et compagnons mentionnés ci-dessus, j'ajouterai les noms de S. Reinach, dont il a déjà été question, d'Antoine Hiéron de Villefosse (qui enseignait l'épigraphie latine et les antiquités romaines à l'École Pratique), de Gaston Maspéro, sur lequel je vais revenir, d'Isidore Lévy, Léon Duvan, etc. ${ }^{39}$.

Cela dit, tandis que F. Cumont était à Paris, à Gand les choses bougeaient. Le 4 novembre 1891, Ch. Michel lui adresse une lettre qui relance l'idée de son entrée à l'Université ${ }^{40}$ : un poste s'est libéré à Liège, qui entraîne le départ de Gand de Ch. Michel lui-même et peut-être aussi de L. Parmentier :

(...) Je viens vous offrir de prendre ma toge à Gand. Vous voyez que tout s'arrange à merveille, sauf que je n'aurais pas le plaisir d'être votre collègue (...). Répondez-moi tout de suite, si possible. Il faut que la campagne soit menée rondement, et il faudra faire agir toutes nos influences parce qu'il se pourrait que certains se missent en campagne pour faire donner la toge à Vercoullie. Mais nous avons les mains pleines d'arguments.

Je n'ai pas besoin de vous dire qu'à Gand le Recteur et l'Admin. seront pour vous.

Je me félicite d'être le premier à vous annoncer cette bonne nouvelle, et de contribuer pour ma part à vous mettre à votre vraie place.

Une lettre de Ch. Michel du 11 novembre nous indique que, cette fois, F. Cumont s'est laissé convaincre; on sent une certaine rétiscence, presque une résignation de sa part face à un projet tant de fois différé, comme s'il avait souhaité rester sa vie durant un « étudiant» :

38 Étonnament, tout en travaillant souvent dans le domaine iranien, F. Cumont n'a en fait jamais reçu de formation linguistique correspondante.

39 Pour ses rapports avec ces différentes personnes, cf. La correspondance, passim.

40 Cette lettre figure dans La correspondance, p. 321-322, et la suivante, p. 322-323. 
Mon cher Ami,

Je n'ai pas besoin de vous dire, n'est-ce pas?, que votre lettre m'a fait un très vif plaisir, et que cette joie a été largement partagée par Thomas et par toute la Faculté. Je souhaite seulement que vous ne vous repentiez pas trop de votre résolution et que vous preniez goût à la tâche que vous acceptez pour nous rendre service (...). Enfin, comme vous le dites, il vous restera toujours le droit, si le métier ne vous sourit pas, de déposer votre toge et de reprendre la vie de l'érudit et de l'archéologue voyageur.

A partir de ce moment, une riche correspondance, dont les auteurs sont surtout Ch. Michel, P. Thomas et M. Cumont père, éclaire les diverses phases de la « campagne » visant à faire entrer F. Cumont à l'Université de Gand avec le titre de Professeur (la « toge »), une campagne qui échoua et obligea ses promoteurs à se contenter en fin de compte (en janvier 1892) d'une nomination de Chargé de Cours, avec la promesse d'une toge imminente (qui arriva dès août 1892 [Professeur extraordinaire], avec l'ordinariat en 1896). A divers égards donc - et aussi à la lumière des épisodes successifs -, le rapport de F. Cumont avec l'Université de Gand, douloureusement interrompu en 1910 suite à une affaire assez trouble ${ }^{41}$, fut dès le début difficile et tendu, comme si F. Cumont n'attendait que l'occasion pour se débarrasser d'une toge qui en définitive lui pesait. Bien plus qu'un Professeur, il a toujours été un savant, un chercheur itinérant.

C'est de Paris que F. Cumont suit toute cette affaire; il fait bien en Belgique les quelques visites que lui conseille Ch. Michel; il fait valoir l'autorité de ses éminents maîtres étrangers, Diels, Mommsen, Duchesne, mais c'est surtout son père qui fait intervenir ses connaissances, des milieux libéraux et laïcs pour l'essentiel ${ }^{42}$. Du reste, les opposants à $F$. Cumont, ou pour le moins les partisans de son concurrent pour l'obtention d'une toge, un certain Vercoullie, proviennent tous des rangs catholiques/cléricaux et aussi flamands / flamingants. Un de ses adversaires, encore que non déclaré, semble avoir été A. De Ceuleneer, le Professeur d'histoire romaine : il avait pourtant été son maître et s'en montrait fier, mais il freina constamment et joua aussi un rôle déterminant dans le départ de F. Cumont de Gand en $1910^{43}$.

L'année 1892 commence par une nouvelle importante : F. Cumont est sur le point d'être nommé Chargé de Cours à l'Université de Gand (la nouvelle paraîtra au Moniteur du 20 janvier). Le 3 janvier, on l'imagine fêtant à la fois cet événement et ses 24 ans. Mais il entend terminer d'abord son semestre parisien : ses charges (histoire politique de l'Orient et de la Grèce, exercices pratiques d'histoire ancienne, encyclopédie de l'histoire ancienne, critique

41 Cf. Appendice 2, dans La Correspondance, p. 495-512.

42 Cela dit, on sait que Marie Faider, la mère de F. Cumont, était croyante et pratiquante; dans une lettre non datée (sans doute de 1891), Émilie Cumont s'inquiète de savoir si Franz fait le carême ou s'il a demandé les dispenses nécessaires, et elle ajoute : « tu sais que maman y tenait beaucoup, fais-le pour elle ».

43

Cf. La correspondance, p. 496-497. 
historique appliquée à l'histoire ancienne) ont été conçues de manière à pouvoir le rendre aussi libre que possible. Ses correspondants l'interrogent régulièrement sur l'avancement de ses recherches sur Julien, sur Philon, sur Mithra, et son maître H. Diels le félicite de sa nomination le 26 janvier 1892 :

Mein lieber Cumont,

Ich sende Ihnen meinen herzlichen Glückwunsch zu Ihrer Ernennung zum Chargé de cours. Ich bedaure es gar nicht, dass Sie Ihre Lieblingsfächer nicht lesen können. Die Technik des Vortrags, die bei Ihnen noch eine grössere Rolle spielen mag als bei uns, lernt sich viel besser an fremden als an vertrauten Gegenständen. Und griechische Geschichte, sapperment, das ist doch keine Kleinigkeit. Das wird ja jedenfalls Ihren übrigen Studien zu gute kommen.

Mi-février, F. Cumont est en tout cas encore à Paris : par l'entremise de B. Haussoullier, il a le privilège de rencontrer le grand Gaston Maspéro ${ }^{44}$. C'est de mars 1892 que date le premier contact épistolaire de F. Cumont avec E. Goblet d'Alviella, titulaire de la chaire d'Histoire des religions à l'Université de Bruxelles ${ }^{45}$, qui le remercie pour l'hommage d'un article et s'intéresse à ses enquêtes mithriaques. En mars encore, Théodore Reinach lui propose de collaborer à la Revue Critique, tandis qu'il prépare une Conférence dans le cadre des cours de Duchesne et d'Haussoullier. Simultanément, les contacts avec ses connaissances germaniques se poursuivent: G. Wissowa se manifeste pour le remercier d'un hommage et de sa participation à la Realenzyklopädie; E. Bormann l'invite à la Journée des Philologues prévue à Vienne en 1893 et l'informe d'un voyage de leur maître Benndorf; $O$. Benndorf lui-même lui adresse ses voux à la fin de l'année 1892 et se félicite de sa collaboration aux Mitteilungen de l'Institut archéologique autrichien; O. Cuntz lui donne des nouvelles de Pernice, de Mommsen et d'Hirschfeld; H. Diels encore lui expose ses recherches en cours et le conseille utilement pour son projet mithriaque («Ihre Idee mit dem Mithrasinventar finde ich sehr gut »).

Le semestre achevé, avant de regagner "définitivement " la Belgique, F. Cumont se rend en Angleterre, à Londres, notamment au British Museum, en compagnie de son maître et ami B. Haussoullier. Parallèlement, il entreprend une réflexion avec $\mathrm{Ch}$. Michel sur le projet de réaliser une «œuvre colossale » (selon l'expression de Ch. Michel dans une lettre du 19 juillet 1892), à savoir un recueil de 600 inscriptions grecques, pour lequel les deux gantois ont sollicité les conseils de S. Reinach, B. Haussoullier et P. Foucart. En août, arrive la nomination de Professeur extraordinaire à Gand, ce dont se réjouit notamment Henri Pirenne.

44 Dans La correspondance, p: 11, 225 et 488, je datais erronément cet événement (mentionné dans des lettres de $B$. Haussoullier et de $M$. Cumont père non datées) de 1891; la meilleure connaissance que j'ai à présent de la chronologie des années de formation de F. Cumont me permet d'être certaine qu'il s'agit de 1892 .

45 À son sujet, cf. La correspondance, p. 217-218. 
C'est dans le dernier trimestre d'octobre 1892 qu'un nouvel intérêt se dessine à travers la lecture de la correspondance : le déchiffrement, l'inventaire et l'exploitation des manuscrits astrologiques, bref ce qui devait conduire à la réalisation, à partir de 1898, du Catalogus codicum astrologorum graecorum. Alessandro Olivieri ${ }^{46}$, en effet, prend contact avec F, Cumont pour divers motifs, notamment pour travailler, sous sa direction, sur les manuscrits astrologiques des bibliothèques italiennes, à commencer par la Laurentienne de Florence. Cette collaboration débouchera sur la participation d'Olivieri aux tomes I, II et IV du Catalogus.

À la fin de l'année 1892, le jeune savant de presque 25 ans qu'était F. Cumont pouvait se vanter d'avoir publié une quinzaine d'articles et compterendus, ainsi que trois livres ou fascicules. Il avait étudié à Gand, Bonn, Berlin, Vienne et Paris; il connaissait Athènes, Rome, Naples, Florence. Il était Professeur extraordinaire de l'Université de Gand et comptait parmi ses «parrains » scientifiques H. Diels, H. Usener, Th. Mommsen, S. Reinach, G. Maspéro, L. Duchesne et bien d'autres encore. Il pouvait faire valoir une très solide formation philologico-historique qu'il allait faire fructifier toute sa vie durant en l'enrichissant et en l'approfondissant sans cesse. Il avait assimilé les enseignements de rigueur, de systématicité, de précision de l'Altertumswissenschaft allemande et ceux de finesse, de nuance, d'ouverture de la science française. Mithra était déjà au cœur de ses préoccupations et de ses recherches, mais il avait fait et faisait de brillantes incursions dans d'autres domaines : l'histoire des idées philosophiques, les questions de transmission des textes, l'épigraphie chrétienne.

En guise de conclusion, je voudrais brièvement attirer l'attention sur l'arrière-plan familial de F. Cumont, tel qu'il apparaît dans sa correspondance. Cette matière ne relève pas directement de la sphère scientifique, mais elle aide, à mon sens, à comprendre comment le tissu social et affectif contribue à l'épanouissement d'une grande personnalité scientifique comme celle qui nous retient ici. Deux figures méritent particulièrement qu'on s'y arrête. D'abord, Charles Faider ${ }^{47}$, le grand-père maternel de F. Cumont, une forte personnalité politique, intellectuelle et morale, qui, durant la période qui nous intéresse, celle du reste de ses dernières années de vie, écrivit douze lettres $^{48}$ à son petit-fils, des lettres pleines d'affection et de sollicitude (« Je t'aime et t'embrasse », écrit-il à la fin de sa lettre du 25 février 1885), mais aussi de conseils sur la manière d'étudier (prendre des notes en lisant, les classer selon une méthode, réciter des textes à haute voix, etc.), sur les fréquentations, bonnes et mauvaises, sur la conduite à suivre (être fort, rangé, économe, calme et solide; dépenser son argent pour des livres, pas pour de la bière), sur la manière de gérer sa carrière scientifique (ne pas prodiguer trop

46 Cf. La correspondance, p. 367-370.

47 Sur Charles Faider (1811-1893), juriste, homme politique et académicien belge de tout premier plan, $c f$. La correspondance, p. 1-2.

48 Les dernières lettres ne sont plus de sa main, car il était devenu aveugle. 
rapidement les résultats de ses recherches). Charles Faider nous apparaît comme un homme de foi : il évoque les « jouissances » que lui vaut la lecture d'une vieille édition de l'«Imitation de Jésus-Christ » ${ }^{49}$, insiste pour que F. Cumont lui procure le Catéchisme de Bossuet et il exprime sa conviction en une forme d'immortalité de l'homme : « il me semble qu'il est impossible que l'on reste indéfiniment séparé absolument de tant d'êtres adorés sans plus connaître leur sort. Mystère qui plonge dans l'Immortalité ! »

Charles Cumont, ensuite, le père de Franz, qui représente pour sa part le courant libéral et progressiste, dans la mesure où l'on perçoit un homme très soucieux des acquis intellectuels de son temps. C'était un industriel du textile, mais aussi un homme qui lisait Fustel de Coulanges, Maeterlinck et Renan, qui suivait avec passion la découverte de la Constitution des Athéniens d'Aristote et regrettait que l'Académie allemande ait préféré Curtius à Mommsen, qui appréciait chaque pas accompli par son fils dans sa carrière d'érudit, jouissant de ses rencontres avec Reinach, Maspéro ou De Ruggiero. Il commande des livres et des revues pour son fils (jusqu'à New York) et les lui fait suivre partout où il est; il s'informe constamment de ses déplacements, de ses projets, et fait régulièrement allusion à sa propre expérience d'homme qui a voyagé à la rencontre de la culture européenne; il se donne beaucoup de mal pour appuyer, à travers un réseau de connaissances politiques, les efforts des Gantois pour "recruter » Franz. Il se montre souvent critique à l'égard de la main mise cléricale sur le monde universitaire belge, mais il exprime des critiques plus dures encore à l'égard du socialisme radical, lorsqu'il relate à son fils des épisodes survenus durant des grèves de houilleurs : il désapprouve ceux qui, parmi les industriels, paient trop peu les ouvriers, mais se dit favorable à une répression dure des grèves. Il tient son fils au courant des choses de la politique (par exemple la réforme du système électoral ou la loi universitaire) et apparaît comme un libéral modéré. C'est un père plein de sollicitude, généreux et capable d'exprimer ses sentiments sans masque : sa préoccupation pour l'évolution de la santé de son beau-père, son désarroi profond après la mort de sa femme. Tout nous indique qu'il constitua un puissant modèle à divers niveaux pour son fils et l'on comprend mieux alors la signification de la dédicace patri carissimo sacrum des Textes et Monuments figurés relatifs aux Mystères de Mithra, la " pierre miliaire » de l'œuvre de Franz Cumont.

Corinne BONNET

Via Valle Scrivia, 37

I - 00141 ROME

49 Sur le rôle que cette lecture a pu jouer dans les derniers jours de vie de F. Cumont, $c f$. La correspondance, p. 49 et n. 152. 


\section{Addendum}

Alors que cet article était déjà déposé, la famille de Cumont m'a confié un nouveau lot d'archives. Parmi ces documents figure une série de lettres envoyées par F. Cumont à ses parents durant ses séjours en Allemagne ( 46 lettres), Grèce (10 lettres) et Italie (18 lettres), entre 1889 et 1891, soit précisément la période prise en considération ici. Le contenu de ces lettres n'infirme en aucun point ma reconstitution des événements; elles apportent bien naturellement un éclairage très intéressant sur la manière dont $\mathrm{F}$. Cumont a vécu cette période de sa vie. Il raconte par exemple sa première rencontre avec Mommsen et Diels; il fait très régulièrement le point sur ses recherches, en particulier sur Mithra (un projet qu'il aborde avec beaucoup de scepticisme); il prend position par rapport aux propositions de l'Université de Gand et exprime explicitement ses réticences devant la perspective d'enseigner; il relate quelques événements dont il a été le témoin, par exemple un défilé de Bismarck et les funérailles de l'impératrice à Vienne, ou encore les grandes fêtes estudiantines de Berlin. Il y est question de ses maîtres : outre Mommsen et Diels (qui lui a proposé de travailler avec lui à Berlin : " mais ça, je m'en gardèrai, porter de l'eau à la rivière »), Hirschfeld, Bormann, Wissowa (qu'il ne semble pas avoir envie de rencontrer), Kekulé, Domazewski, etc. Ses impressions de Grèce et d'Italie sont particulièrement intéressantes : description de l'Acropole, d'Éleusis, d'Argos, de l'Étolie et de l'Acarnanie, de Rome découverte sous la neige !-, d’Ostie et Pompéi, de Florence, Sienne, Bologne, etc., avec de nombreux détails sur les conditions matérielles de ses excursions. Bien entendu, ces lettres traitent aussi de diverses questions familiales, économiques et politiques. Il va de soi que ces documents seront publiés in extenso dans un proche avenir. 